\title{
Prevalencia de Bruxismo y Estrés en Estudiantes de Odontología de la Pontificia Universidad Católica de Chile
}

\author{
Bruxism and Stress Prevalence in Dentistry Students \\ of the Pontificia Universidad Católica de Chile
}

\author{
Kristine von Bischhoffshausen P. ${ }^{1}$; Andrea Wallem H. ${ }^{2}$; Alfonso Allendes A. ${ }^{1}$ \& Rodrigo Díaz M. ${ }^{3}$
}

VON BISCHHOFFSHAUSEN, P. K.; WALLEM, H. A.; ALLENDES, A. A. \& DíAZ, M. R. Prevalencia de bruxismo y estrés en estudiantes de odontología de la Pontificia Universidad Católica de Chile. Int. J. Odontostomat., 13(1):97-102, 2019.

RESUMEN: El bruxismo corresponde a una actividad parafuncional de apretamiento dentario por acción de los músculos masticatorios. Dentro de los factores etiológicos del bruxismo, se encuentran estados emocionales como la ansiedad, depresión y situaciones de estrés, entre otros. Los estudiantes de Odontología, especialmente aquellos que se encuentran en su ciclo clínico, se someten a situaciones de alta exigencia que les generan estados de depresión, estrés y ansiedad. El objetivo de este estudio es medir la prevalencia de trastornos psicológicos como depresión, ansiedad y estrés y bruxismo en estudiantes de Odontología de 4to y 5 to año de la Pontificia Universidad Católica de Chile. Mediante el uso de cuestionarios y exámenes clínicos, utilizando el cuestionario de la Asociación Americana de Medicina del Sueño y la Escala de Depresión-Ansiedad y Estrés (DAS-21), se determinó que el $62 \%$ de los estudiantes presentaron bruxismo, con predominio en mujeres. Todos los alumnos que presentaron bruxismo manifestaron niveles de depresión, ansiedad y estrés. Estas cifras son importantes a considerar como señal de alerta y para generar medidas preventivas y terapéuticas para disminuir el bruxismo y los trastornos psicológicos asociados.

PALABRAS CLAVE: bruxismo, estudiantes, odontología, estrés.

\section{INTRODUCCIÓN}

Dentro de las actividades de los músculos masticatorios existen dos tipos; las funcionales que permiten realizar actividades sin generar daño al sistema estomatognático (SE), y las parafuncionales que corresponden a una hiperactividad neuromuscular repetitiva e inconsciente que genera sobrecarga sobre las estructuras estomatognáticas (Romo, 2011). La actividad parafuncional más común es el bruxismo, que consiste en el movimiento parafuncional e involuntario rítmico de apriete, rechinamiento, trituración y/o masticación (en ausencia de alimento) que puede llevar a una disfunción de la musculatura masticatoria, generando un trauma constante y crónico sobre las estructuras del SE (Romo). El bruxismo puede producirse durante el día en estado de vigilia o de noche durante el sueño; no se relaciona con la luz solar, si no con el ciclo de sueño (Nissani, 2001). Actualmente existe una gran variabilidad en la prevalencia del bruxismo, llegando incluso a alcanzar un $90 \%$ en algunas poblaciones, la mayor incidencia es entre los 20 y 50 años de edad, siendo necesario su tratamiento en personas con sintomatología (Nissani).

La relación entre bruxismo y trastornos temporomandibulares (TTM) ha sido frecuentemente estudiada, determinando si esta parafunción conlleva un factor de riesgo para padecer algún TTM. Los TTM son considerados como un subgrupo de los trastornos que afectan al sistema músculo-esquelé-

\footnotetext{
${ }^{1}$ Pontificia Universidad Católica de Chile, Santiago, Chile.

2 Escuela de Odontología, Facultad de Medicina. Pontificia Universidad Católica de Chile, Santiago, Chile.

${ }^{3}$ Facultad de Medicina Pontificia Universidad Católica, Escuela de Odontología, Internado Intramural de Oclusión y Trastornos Temporomandibulares, Santiago, Chile.
} 
VON BisChHOffShauSen, P. K.; WALLEM, H. A.; ALLENDES, A. A. \& DíAZ, M. R. Prevalencia de bruxismo y estrés en estudiantes de odontología de la Pontificia Universidad Católica de Chile. Int. J. Odontostomat., 13(1):97-102, 2019.

tico generalmente relacionados con sobrecarga, malos hábitos, alteraciones posicionales, con una respuesta inadecuada o insuficiente de los tejidos afectados (Shetty et al., 2010). Por lo tanto el bruxismo no se considera como un trastorno o desorden per se, ya que no correspondería a una condición que produce daños inherentemente, más bien se considera como un factor de riesgo con consecuencias negativas en la salud oral en ciertos individuos (Lobbezoo et al., 2018). El bruxismo produce sobrecarga sobre las estructuras, produciendo alteraciones a nivel articular, dentario, periodontal y neuromuscular. A medida que estas no logran adaptarse, se produciría una disfunción o trastorno temporomandibular (Romo).

La etiología del bruxismo es multifactorial y se ha relacionado con la maloclusión, hábitos orales, trastornos temporomandibulares y a estados emocionales alterados (Molina et al., 2001). Se ha demostrado que el desarrollo de hábitos nerviosos manifestados en tensión muscular se presentan como mecanismo de expulsión de ansiedad (Okeson, 2008). También se ha postulado la relación existente entre el bruxismo y ciertos factores psicosociales como agresión, hiperactividad, ansiedad y necesidad de autocontrol (Molina et al.). Los factores psicosociales consisten en factores individuales e interpersonales que tienen un impacto en la capacidad de adaptación del paciente; aquellos que presentan TTM pueden tener una personalidad o condición emocional que dificulte la resolución de las situaciones cotidianas del día a día. Algunos pacientes con TTM experimentan más ansiedad que los pacientes sanos, siendo los síntomas y signos clínicos una manifestación de un problema de estrés emocional o trastornos psicológicos (Lobbezoo et al.).

Se ha propuesto la realización de un estudio de tipo descriptivo de prevalencia, para evaluar así la magnitud y la distribución de bruxismo y su relación con el nivel de estrés y ansiedad en los alumnos de 4to y 5 to año de la carrera de Odontología de la Facultad de Medicina de la Pontificia Universidad Católica de Chile. Hemos elegido estos cursos (4to y 5to año), ya que son aquellos en donde se produce un cambio importante en los niveles de ansiedad y de estrés de los alumnos; corresponde a la entrada al ciclo clínico, en el cual se realiza la atención directa de pacientes y es donde se produce el mayor porcentaje de deserción y congelamiento de la carrera. Obtenidos los resultados, se comparará con estudios similares.

\section{MATERIAL Y MÉTODO}

Se aplicó un cuestionario, la escala abreviada de depresión-ansiedad y estrés (DASS-21) (Daza et al., 2002) (Tabla I), para determinar el nivel de estrés a los alumnos, la cual se aplicó a todos los alumnos que estaban cursando el $4^{\circ}$ y $5^{\circ}$ año de la carrera de Odontología en la Pontificia Universidad Católica de Chile en el año $2017(n=127)$.

Para determinar la presencia de bruxismo se utilizaron los criterios de la Asociación Americana de Medicina de Sueño (AASM), en relación con criterios anamnésicos y clínicos, que incluyen los siguientes 10 parámetros (Tabla II): reporte de apriete y/o rechinamiento dentario, historia reciente de apriete reportado por un amigo, parientes y/o pareja, dolor o sensación de cansancio en los músculos de la cara, historia reciente (últimos 6 meses) de pérdida o fractura de restauraciones, coronas, puentes $u$ otros, sin causa aparente, sensación de poseer los dientes desgastados, dolor muscular a la palpación, presencia de bruxofacetas, hipertrofia de músculos maseteros y temporal, movilidad dentaria aumentada asociada con facetas de desgaste dentario y pérdida y/o fractura de restauraciones sin causa aparente (American Academy of Sleep Medicine, 2014). Cada uno de estos parámetros entrega una puntuación de 1 punto, y al obtener un puntaje mayor a 3 puntos se constata la presencia de bruxismo.

Los exámenes fueron realizados por los alumnos del Internado en Oclusión y Trastornos Temporomandibulares de la misma casa de estudios del año 2017. Por último, los resultados fueron analizados como variables independientes.

\section{RESULTADOS}

Con respecto a la presencia de bruxismo entre los estudiantes, según los parámetros de la AASM, de un total de 127 alumnos (72 alumnos de 4 to y 55 alumnos de 5to año), 79 alumnos demostraron tener bruxismo correspondiente al $62,2 \%$ del total (puntaje mayor a 3$)$, teniendo 31 alumnos bruxismo leve $(39,2$ $\%$ ) (puntaje entre 3 y 4 ), 46 alumnos bruxismo moderado $(58,2 \%$ ) (puntaje entre 5 y 7 ) y 2 alumnos bruxismo severo $(2,5 \%)$ (puntaje entre 8 y 10) (Fig. 1).

Según el sexo, la distribución de bruxismo es 
mayor en el género femenino. Se constató que 65 mujeres presentan bruxismo siendo un $82 \%$ del total de bruxómanos, 27 alumnas presentaron bruxismo leve $(41,5 \%), 36$ alumnas bruxismo moderado (55 $\%)$ y 2 alumnas bruxismo severo (3\%). El número de hombres que presentaron bruxismo fue de 14 alumnos siendo el $17,7 \%$ del total de bruxómanos, 4 alumnos presentaron bruxismo leve $(28,5 \%), 10$ alumnos bruxismo moderado $(71,4 \%)$ y 0 alumnos bruxismo severo $(0 \%)$.

En el cuestionario de la escala abreviada de Depresión-Ansiedad y Estrés (DASS-21) (Tabla I) se reportaron los resultados según severidad de cada factor: normal, leve, moderado, severo y muy severo. En este caso fueron contabilizadas las respuestas única- mente de los alumnos que presentaron bruxismo (79 personas).

Los resultados sobre el factor depresión demostraron 15 respuestas en el nivel normal, 6 en leve, 16 en moderado, 17 en severo y 25 en muy severo. En el factor ansiedad hubo 7 respuestas en el nivel normal, 8 en leve, 7 en moderado, 15 en severo y 42 en muy severo. En el factor estrés hubo 22 respuestas en el nivel normal, 13 en leve, 12 en moderado, 14 en severo y 17 en muy severo.

Podemos percatarnos de que los niveles con mayor número de respuesta son : depresión muy severa, ansiedad muy severa, stress normal y stress muy severo (Tabla III).

Tabla I. Escala abreviada de depresión-ansiedad y estrés (DASS-21).

\begin{tabular}{|c|c|c|c|c|c|c|c|c|}
\hline \multicolumn{9}{|c|}{$\begin{array}{l}\text { Por favor lea las siguientes afirmaciones } \\
\text { La éscala de čalificáción es lä śiguiente: }\end{array}$} \\
\hline 0 & \multicolumn{8}{|l|}{ No me aplicó } \\
\hline 1 & \multicolumn{8}{|c|}{ Me aplicó un poco, o durante parte del tiempo } \\
\hline 2 & \multicolumn{8}{|c|}{ Me aplicó bastante, o durante una buena parte del tiempo } \\
\hline \multirow[t]{2}{*}{3} & \multicolumn{8}{|c|}{ Me aplicó mucho, o la mayor parte del tiempo. } \\
\hline & normal & leve & moderada & severa & \multicolumn{4}{|c|}{ muy severa } \\
\hline \multicolumn{2}{|c|}{ Depresión } & $10-13$ & $14-20$ & $21-27$ & \multicolumn{4}{|c|}{$28+$} \\
\hline \multicolumn{2}{|c|}{ Ansiedad } & $8-9$ & $10-14$ & $15-19$ & \multicolumn{4}{|c|}{$20+$} \\
\hline Estrés & $0-14$ & $15-18$ & $19-25$ & $26-33$ & \multicolumn{4}{|c|}{$34+$} \\
\hline 1. & \multicolumn{4}{|l|}{ Me costó mucho relajarme. } & 0 & 1 & 2 & 3 \\
\hline 2. & \multicolumn{4}{|c|}{ Me di cuenta que tenia la boca seca. } & 0 & 1 & 2 & 3 \\
\hline 3. & \multicolumn{4}{|c|}{ No podía sentir ningún sentimiento positivo. } & 0 & 1 & 2 & 3 \\
\hline 4. & \multicolumn{4}{|l|}{ Se me hizo difícil respirar. } & 0 & 1 & 2 & 3 \\
\hline 5. & \multicolumn{4}{|c|}{ Se me hizo difícil tomar la iniciativa para hacer cosas. } & 0 & 1 & 2 & 3 \\
\hline 6. & \multicolumn{4}{|c|}{ Reaccioné exageradamente en ciertas situaciones. } & 0 & 1 & 2 & 3 \\
\hline 7. & \multicolumn{4}{|c|}{ Sentí que mis manos temblaban. } & 0 & 1 & 2 & 3 \\
\hline 8. & \multicolumn{4}{|l|}{ Sentí que tenia muchos nervios. } & 0 & 1 & 2 & 3 \\
\hline 9. & \multicolumn{4}{|c|}{$\begin{array}{l}\text { Estaba preocupado por situaciones en las cuales podía tener pánico o en las } \\
\text { que podría hacer el ridículo. }\end{array}$} & 0 & 1 & 2 & 3 \\
\hline 10. & \multicolumn{4}{|c|}{ Sentí que no tenia nada por que vivir. } & 0 & 1 & 2 & 3 \\
\hline 11. & \multicolumn{4}{|l|}{ Noté que me agitaba. } & 0 & 1 & 2 & 3 \\
\hline 12. & \multicolumn{4}{|l|}{ Se me hizo difícil relajarme. } & 0 & 1 & 2 & 3 \\
\hline 13. & \multicolumn{4}{|l|}{ Me sentí triste y deprimido. } & 0 & 1 & 2 & 3 \\
\hline 14. & \multicolumn{4}{|c|}{ No toleré nada que no me permitiera continuar con lo que estaba haciendo. } & 0 & 1 & 2 & 3 \\
\hline 15. & \multicolumn{4}{|c|}{ Sentí que estaba al punto de pánico. } & 0 & 1 & 2 & 3 \\
\hline 16. & \multicolumn{4}{|l|}{ No me pude entusiasmar por nada. } & 0 & 1 & 2 & 3 \\
\hline 17. & \multicolumn{4}{|c|}{ Sentí que valía muy poco como persona. } & 0 & 1 & 2 & 3 \\
\hline 18. & Sentí que estaba muy irritable. & & & & 0 & 1 & 2 & 3 \\
\hline 19. & $\begin{array}{l}\text { Sentí los latidos de mi corazón } \\
\text { físico. }\end{array}$ & r de no $h$ & echo ningún & erzo & 0 & 1 & 2 & 3 \\
\hline 20. & Tuve miedo sin razón. & & & & 0 & 1 & 2 & 3 \\
\hline 21. & Sentí que la vida no tenía ningú & ido. & & & 0 & 1 & 2 & 3 \\
\hline
\end{tabular}


Tabla II. Parámetros utilizados para determinar prevalencia de bruxismo

\begin{tabular}{lll}
\hline Criterios Anamnésicos & Sí & No \\
\hline 1. & Reporte de apriete y/o rechinamiento dentario. \\
2. Historia reciente de apriete y/o rechinamiento dentar io reportado por un & \\
amigo, pariente y/o pareja. & \\
3. Dolor o sensación de cansancio en los músculos de la cara. & \\
4. Historia reciente (últimos 6 meses) de pérdida o fractura de restauraciones, & No \\
coronas, puentes u otros, sin una causa aparente. & Sí \\
5. Sensación de poseer los dientes desgastados. & \\
\hline \multicolumn{2}{l}{ Criterios Clínicos } & \\
\hline 1. Dolor muscular a la palpación. & \\
2. Presencia de bruxofacetas & \\
3. Hipertrofia de los músculos Masetero y Temporal (simétrica o asimétrica) \\
4. Movilidad dentaria aumentada asociada con facetas de desgaste dentario \\
5. Pérdida y/o fractura de restauraciones sin causa aparente
\end{tabular}

Cada respuesta afirmativa tendrá un valor equivalente a 1 punto y cada respuesta negativa 0 puntos. Los criterios 1 y 2 serán considerados como principales. Para que un paciente sea diagnosticado como bruxista debe presentar 2 criterios anamnésicos y 1 criterio clínico. Dentro de los criterios anamnésicos debe estar presente uno de los criterios principales. De esta forma, los pacientes con 3 o 4 puntos serán clasificados como bruxistas leves, los pacientes con 5 a 7 puntos como bruxistas moderados y los pacientes con 8 a 10 puntos como bruxistas severos. $\mathrm{Si}=1 \mathrm{No}=0$ 3 a $4=$ Leve 5 a $7=$ Moderado 8 a $10=$ Severo.

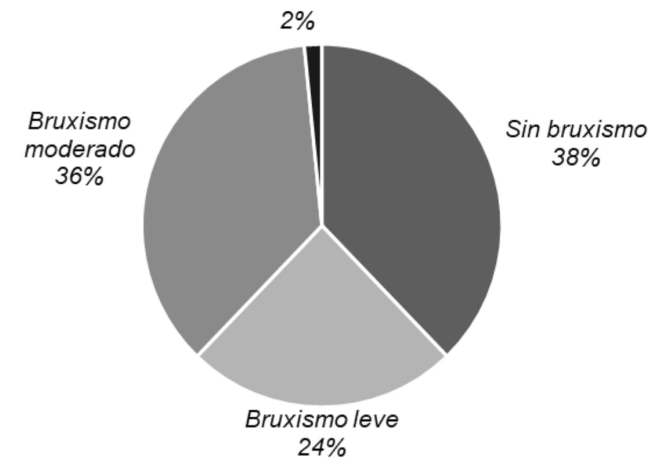

Fig 1. Prevalencia de bruxismo según nivel de severidad.
Para relacionar nivel de bruxismo con niveles de depresión, ansiedad y estrés se observa que en el nivel de depresión muy severo $(\mathrm{n}=25)$ tenemos 8 alumnos con bruxismo leve, 16 con moderado y 1 con severo. En ansiedad muy severa $(n=42)$ se encontramos 15 alumnos con bruxismo leve, 26 con moderado y 1 con severo. En estrés muy severo $(n=17)$ hubo 6 alumnos con bruxismo leve, 10 con moderado y 1 con severo, el $100 \%$ de los alumnos con estrés muy severo presenta algún grado de bruxismo (Fig. 2).

\section{DISCUSIÓN}

Tabla III. Niveles de respuesta.

\begin{tabular}{ll}
\hline Depresión & 64 \\
Leve & 6 \\
Moderado & 16 \\
Severo & 17 \\
Muy severo & 25 \\
Ansiedad & 72 \\
Leve & 8 \\
Moderado & 7 \\
Severo & 15 \\
Muy severo & 42 \\
Estrés & 56 \\
Leve & 13 \\
Moderado & 12 \\
Severo & 14 \\
Muy severo & 17 \\
\hline
\end{tabular}

Estos datos nos permiten evaluar la prevalencia de estrés y bruxismo en los alumnos de Odontología cumpliendo así los objetivos propuesto en el trabajo de investigación.

Se ha demostrado en diversos estudios la asociación existente entre los niveles de depresión, angustia y estrés y el bruxismo. Un estudio de Kampe comparó aspectos de la personalidad de pacientes con bruxismo y sin bruxismo. Se observó que los sujetos bruxómanos tenían mayor predisposición a la ansiedad, eran más vulnerables a desórdenes psicosomáticos y tenían mayores problemas de sociabilización (Kampe et al., 1997). Así también otros estudios han demostrado que facto- 


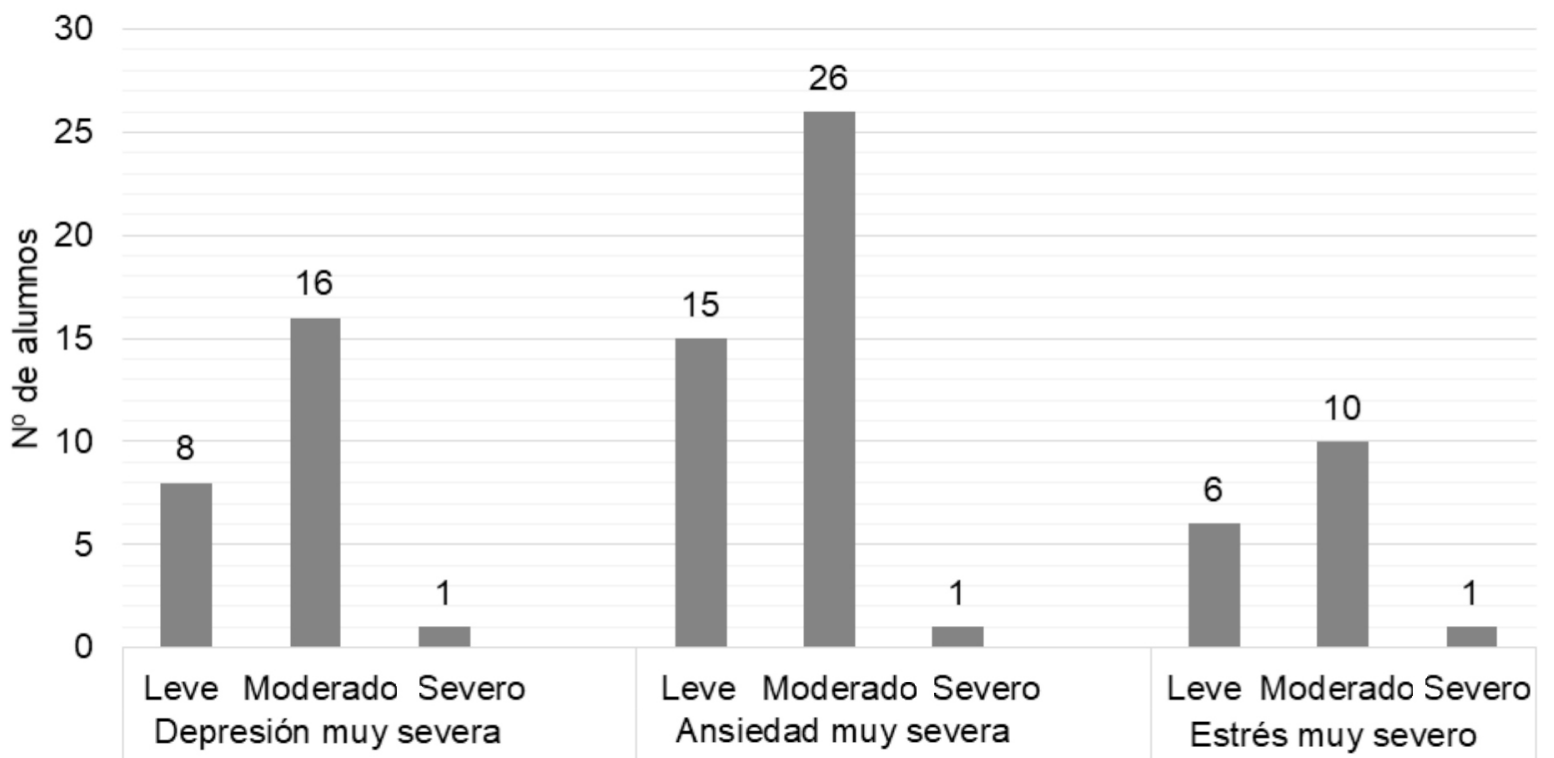

Fig. 2. Prevalencia de trastornos de depresión, ansiedad y estrés en alumnos con bruxismo de 4to y 5to de odontología, de la Pontificia Universidad Católica de Chile.

res psicológicos o emocionales como ansiedad, estrés, miedo y frustración se relacionan estrechamente con la hiperactividad muscular, sugiriendo que podrían participar en la etiología del bruxismo como un factor promotor de esta parafunción (Bader \& Lavigne, 2000).

Se han realizado este tipo de investigaciones a nivel mundial con el fin de relacionar el bruxismo con los factores psicosociales y conductuales en estudiantes universitarios. En un estudio realizado en estudiantes japoneses de Odontología se observó una asociación positiva entre la cantidad de consumo de alcohol y el estado de agresión- hostilidad con el bruxismo del sueño (Flores et al., 2003). Otro estudio realizado en la Universidad de Ciencias de la Salud de Lituania demostró que la prevalencia de estrés es casi la misma que la prevalencia de reflujo gastroesofágico, enfermedades periodontales y bruxismo, siendo este último signo detectado con una asociación estadísticamente significante (Arman et al., 2016). Es posible afirmar que existe una alta prevalencia de bruxismo en estudiantes, consistente con la literatura científica disponible; se describen alteraciones neuromusculares inducidas por estrés, aumentando también la prevalencia de desórdenes Temporomandibulares (Cavallo et al., 2016). Se ha demostrado asociación entre estrés y bruxismo, sobre todo en estudiantes universitarios, con un aumento en la incidencia de bruxismo du- rante las últimas décadas con cifras de hasta $83 \%$ en estudiantes de odontología (Quadri et al., 2015). Con respecto a los resultados obtenidos, la importante cifra de alumnos que presentan bruxismo de moderado a severo, es una señal de alerta en cuanto a la salud oral debido a que se presentan desgastes dentarios, pérdida de restauraciones/dientes, y dolor muscular.

A partir de la evidencia disponible a nivel mundial sobre la percepción de bruxismo y estrés en estudiantes podemos reforzar la idea de que existe una asociación, claramente observable en los resultados. En el estudio de Moncada en estudiantes chilenos se observó clínicamente que, en la muestra de bruxómanos, predominaban el estrés, la tensión y la ansiedad como estados recurrentes, asociados a altas exigencias académicas (Moncada et al., 2009). A partir de las observaciones y resultados se concluyó que el desarrollo de conductas asertivas, expresiones emocionales, entrenamiento de conductas interpersonales y manejo de estrés serían fundamentales en el tratamiento del bruxismo (Moncada et al.). Por lo tanto, el estudio de estas cifras permitirá establecer conductas y métodos pedagógicos para en un futuro mejorar la situación de los estudiantes y así prevenir posibles las patologías asociadas tanto a trastornos psicológicos como trastornos parafuncionales del sistema estomatognático. 
VON BISCHHOFFSHAUSEN, P. K.; WALLEM, H. A.; ALLENDES, A. A. \& DÍAZ, M. R. Bruxism and Stress Prevalence in Dentistry Students of the Pontificia Universidad Católica de Chile. Int. J. Odontostomat., 13(1):97-102, 2019.

ABSTRACT: Bruxism corresponds to a parafunctional activity of dental tightening due to the action of masticatory muscles. Within the etiological factors of bruxism, there are emotional states such as anxiety, depression and stress situations, among others. Dental students, especially those who are in their clinical cycle, are subjected to high demand situations that generate states of depression, stress and anxiety. The aim of this study is to measure the prevalence of psychological disorders such as depression, anxiety and stress and bruxism in 4th and 5th year of dentistry students of the Pontificia Universidad Católica de Chile. Through the use of questionnaires and clinical examinations, using the questionnaire of the American Association of Sleep Medicine and the Depression-Anxiety and Stress Scale (DAS-21), it was determined that $62 \%$ of the students presented bruxism, with predominance in women. All of the students that presented bruxism, manifested levels of depression, anxiety and stress. These numbers are important to consider as a warning signal and to generate preventive and therapeutic measures that contribute to the reduction of bruxism and associated psychological disorders.

KEY WORDS: bruxism, students, dentistry, stress.

\section{REFERENCIAS BIBILIOGRÁFICAS}

American Academy of Sleep Medicine. International Classification of Sleep Disorders. $3^{\text {rd }}$ ed. Westchester, American Academy of Sleep Medicine, 2014. pp.303-11.

Arman, K.; Petruninaite, A.; Grigalauskiene, R. \& Slabsinskiene, E. Stress experience and effect on self-perceived oral health status among high school students. Stomatologija, 18(3):759, 2016.

Bader, G. \& Lavigne, G. Sleep bruxism; an overview of an oromandibular sleep movement disorder. Sleep Med. Rev., 4(1):27-43, 2000

Cavallo, P.; Carpinelli, L. \& Savarese, G. Perceived stress and bruxism in university students. BMC Res. Notes, 9(1):514, 2016.

Daza, P; Novy, D. M.; Stanley, M. A. \& Averill, P. The Depression Anxiety Stress Scale-21: Spanish Translation andValidation with a Hispanic Sample. J. Psychopathol. Behav. Assess., 24(3):195-205, 2002.

Flores, R. I. G.; Baba, K.; Haketa, T.; Sasaki, Y.; Kino, K. \& Ohyama, T. Risk Factors for sleep bruxism in Japanese dental students. J. Appl. Res., 3(4):420-8, 2003.

Kampe, T.; Tagdae, T.; Bader, G.; Edman, G. \& Karlsson, S. Reported symptoms and clinical findings in a group of subjects with longstanding bruxing behaviour. J. Oral Rehabil., 24(8):581-7, 1997.

Lobbezoo, F.; Ahlberg, J.; Raphael, K. G.; Wetselaar, P.; Glaros, A. G.; Kato, T.; Santiago, V.; Winocur, E.; De Laat, A.; De Leeuw, R.; Koyano, K.; Lavigne, G. J.; Svensson, P. \&
Manfredini, D. International consensus on the assessment of bruxism: Report of a work in progress. J. Oral Rehabil., 45(11):837-44, 2018.

Molina, O. F.; dos Santos, J.; Mazzetto, M.; Nelson, S.; Nowlin, T. \& Mainieri, E. T. Oral jaw behaviors in TMD and bruxism: a comparison study by severity of bruxism. Cranio, 19(2):11422, 2001.

Moncada, A. L; Gallardo, R. I.; Aguilar, M. L.; Conejan, N. C.; Dreyer, A. E. \& Rojas, A. G. Bruxómanos presentan un estilo de personalidad distintivo. Rev. Chil. Neuro-Psiquiatr., 47(2):105-13, 2009.

Nissani, M. A bibliographical survey of bruxism with special emphasis on non-traditional treatment modalities. J. Oral Sci., 43(2):73-83, 2001.

Okeson, J. P. Tratamiento de Oclusión y Afecciones Temporomandibulares. 6a ed. Barcelona, Elsevier, 2008.

Quadri, M. F. A.; Mahnashi, A.; Almutahhir, A. A.; Tubayqi, H.; Hakami, A.; Arishi, M. \& Alamir, A. Association of awake bruxism with khat, coffee, tobacco, and stress among Jazan University students. Int. J. Dent., 2015:842096, 2015.

Romo, F. Tópicos de Odontología Integral. Santiago de Chile, Universidad de Chile, Facultad de Odontología, 2011.

Shetty, S.; Pitti, V.; Satish Babu, C. L.; Surendra Kumar, G. P. \& Deepthi, B. C. Bruxism: a literature review. J. Indian Prosthodont. Soc., 10(3):141-8, 2010.

Dirección para correspondencia:

Kristine von Bischhoffshausen P.

Escuela de Odontología

Facultad de Medicina.

Pontificia Universidad Católica de Chile

Avenida Vicuña Mackenna 4860-Macúl

Santiago

CHILE

Email: kvvonbischhoffshause@uc.cl

Recibido: 23-10-2018

Aceptado: 07-12-2018 